\title{
In vitro Adsorption of Paraquat onto Substituted Chitosan Beads
}

\author{
Takeo NAKAMURA*1, Syojiro KYOTANI*1, Naohito KAWASAKI*1, \\ Seiki TANADA*1 and Yutaka NISHIOKA*2 \\ ${ }^{* 1}$ Faculty of Pharmaceutical Sciences, Kinki University, Osaka \\ *2 Department of Pharmacy, Kochi Medical School Hospital, Kochi
}

\begin{abstract}
Paraquat removal by chitosan with different substituents was investigated in vitro for evaluation of chitosan as a paraquat adsorbent in emergency treatment for acute paraquat poisoning.

Chitosan $\mathrm{CM}$ and $\mathrm{SU}$, in which the hydroxyl group at the $\mathrm{C}_{6}$ position is replaced by a carboxymethyl and sulfonic group, respectively, removed paraquat abundantly in purified water. However an inhibitory effect of sodium chloride on paraquat removal by $\mathrm{CM}$ and $\mathrm{SU}$ was recognized.

On the other hand, paraquat removal by cross-linked chitosan (BCW) was accelerated in saline solutions. It was recognized that more paraquat was removed by BCW chitosan when the sodium chloride concentration was less than $1.8 \%$.

It was confirmed that some chitosan beads (CM and SU) could function as effective paraquat adsorbents through in vitro experiments.
\end{abstract}

Key words : Paraquat poisoning, Chitosan, Adsorbent, Sodium chloride addition

\section{Introduction}

Paraquat $\left(1,1^{\prime}\right.$ - dimethyl-4-4' - bipyridylium chloride) is widely used as a non-hormone, nonselective herbicide, and its amount of utilization has increased year by year because of its superior herbicidal effect ${ }^{1)}$. Paraquat is unlikely to produce serious problems when properly used; however, many people have died through accidental, suicidal or homicidal ingestion of it $^{2,3)}$. The mortality from paraquat poisoning is higher than that from any other type of poisoning associated with agricultural chemicals ${ }^{1)}$.

Emergency treatment for paraquat poisoning involves methods which minimize the internal absorption by excretion of paraquat through the digestive tract and methods which remove paraquat in the blood. The former method involves gastrointestinal lavage and the administration of a purgative ${ }^{4}$. The latter utilizes activated carbon hemoperfusion, with reported survival cases ${ }^{4}$. On the other hand, a method which hinders the absorption of paraquat by adsorbents has been proposed. As adsorbents, the effectiveness of aluminium silicate ${ }^{5)}$, bentonite ${ }^{6)}$, activated carbon ${ }^{4)}$ and cation-exchange resin ${ }^{7 \sim 9)}$, have been reported. However, no specific and effective antidote for paraquat poisoning is available at present.

Recently, chitin and chitosan have drawn great interest from the standpoint of utilization of natural resources. Chitin, $\beta$ - (1-4) -poly-Nacetyl-D-glucosamine, as well as its deacetylated derivative chitosan, is widely and abundantly distributed in shells of crustaceans and insects, organs of molluscs, and cell walls of fungi and

Reprint requests to : Takeo Nakamura, Faculty of Pharmaceutical Sciences, Kinki University, Kowakae

3-4-1, Higashi-Osaka, Osaka 577, Japan 
bacteria. Chitin and chitosan have extremely low toxicity, and are biocompatible. Furthermore, chemical modification of chitin and chitosan is easy ${ }^{10)}$.

Most studies carried out using these biopolymers have dealt with water treatment ${ }^{11 \sim 13)}$, or medical and pharmaceutical fields ${ }^{14 \sim 16)}$, and little work on the application of chitin and chitosan as antidotes for paraquat poisoning has been done so far.

In this investigation, we use substituted chitosan beads and discuss paraquat removal for the purpose of emergency treatment for acute paraquat poisoning in vitro.

\section{Materials and Methods}

Paraquat was purchased from Wako Pure Chemical Industry Ltd., and its grade was for the testing of agricultural chemical residues. Four kinds of substituted chitosan beads (CM, $\mathrm{SU}, \mathrm{CS}$ and BCW) made by Fuji Spinning Co.
Ltd. were obtained from Wako Pure Chemical Industry Ltd. , and their particle sizes were 4270 mesh. They have different substituted groups in their chemical structures as shown in Figure 1. Activated carbon (BAC-MUL) was obtained from Kureha Chemical Ltd. , and its particle sizes were 32-48 mesh.

The time courses of amounts of paraquat adsorbed onto chitosan were measured with a paraquat solution/chitosan system at $37^{\circ} \mathrm{C}$. One gram of chitosan or activated carbon was added to $100 \mathrm{ml}$ of a stirred paraquat solution $(300 \mathrm{rpm}$, $c a .800 \mathrm{mg} / 1)$ and the paraquat concentrations in the suspension were measured at regular intervals. The paraquat concentration was measured by a colorimetric method with an alkaline sodium dithionite solution ${ }^{17)}$. Paraquat solution was diluted with purified water, artificial gastric juice ${ }^{18)}$, which contained $2.4 \mathrm{~g}$ of sodium chloride and $24.0 \mathrm{ml}$ of diluted hydrochloride (10\%) in 1000 $\mathrm{ml}$ or normal saline solution $(0.9 \%$ sodium chlo-

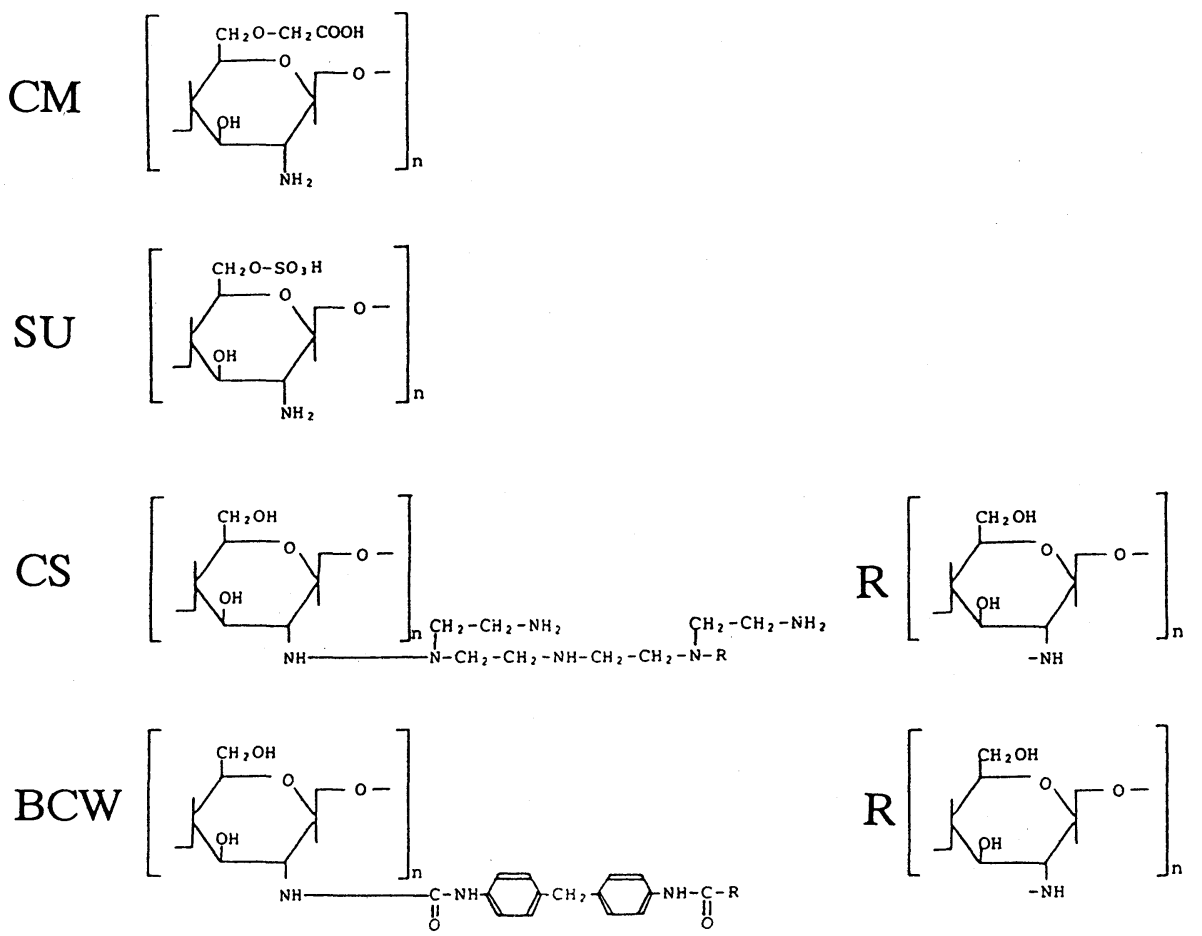

Fig. 1 Chemical structures of chitosan beads 
ride). Furthermore, the time courses of amounts of paraquat adsorbed onto chitosan beads in various saline concentrations were measured.

The removal ratio of paraquat was calculated by the following formula; $\left[\left(\mathrm{C}_{0}-\mathrm{Ct}\right) / \mathrm{C}_{0}\right] \times 100$, where $\mathrm{C}_{0}$ is the initial concentration of paraquat and $\mathrm{Ct}$ is the paraquat concentration at an elapsed time of $60 \mathrm{~min}$.

\section{Results}

The removal ratio for paraqut after $60 \mathrm{~min}$ is indicated in Table 1. The removal ratios with $\mathrm{CM}$ and SU in purified water and artificial gastric juice were higher than those with activated carbon. In normal saline solution, however, the removal ratios of chitosan beads were lower than

Table 1 Paraquat removal ratios of chitosan beadsand activated carbon

\begin{tabular}{lrcc}
\hline \multirow{2}{*}{\multicolumn{1}{c}{ adsorbents }} & \multicolumn{3}{c}{ removal ratio (\%) } \\
\cline { 2 - 4 } $\mathrm{H}_{2} \mathrm{O}^{*}$ & $\mathrm{HCl}^{* *}$ & $\mathrm{NaCl}^{* * *}$ \\
\hline chitosan bead CM & 97.0 & 80.9 & 26.1 \\
chitosan bead $\mathrm{SU}$ & 74.1 & 43.9 & 21.0 \\
chitosan bead CS & 9.9 & 5.3 & 9.3 \\
chitosan bead BCW & 3.6 & 11.1 & 13.0 \\
activated carbon & 16.9 & 28.5 & 45.1 \\
\hline
\end{tabular}

$*:$ in purified water, **: in artificial gastric juice, *** : in normal saline solution that of activated carbon.

Figure 2 shows the time courses of amounts of paraquat adsorbed onto chitosan beads and activated carbon in purified water, artificial gastric juice and normal saline solution. In purified water, the amounts adsorbed onto $\mathrm{CM}$ and SU were abruptly increased at an elapsed time of 30 min and then increased slowly. The increases of the amounts of paraquat adsorbed onto CS and BCW were small, and the values at each elapsed time were smaller than that onto activated carbon. In artificial gastric juice, the amount of paraquat adsorbed onto $\mathrm{CM}$ was greatly increased at $40 \mathrm{~min}$ and then increased slowly. The amount adsorbed onto chitosan beads was lower in artificial gastric juice than in purified water except in the case of BCW. In normal saline solution, the amounts adsorbed onto CM and SU were smaller than in purified water and in artificial gastric juice. In normal saline solution, the amount of paraquat adsorbed onto activated carbon was larger than that adsorbed onto chitosan beads. The paraquat removal by CM and SU was more effective in purified water than in artificial gastric juice and/or in normal saline solution . In contrast, the degrees of increases in the amounts of paraquat adsorbed onto BCW and
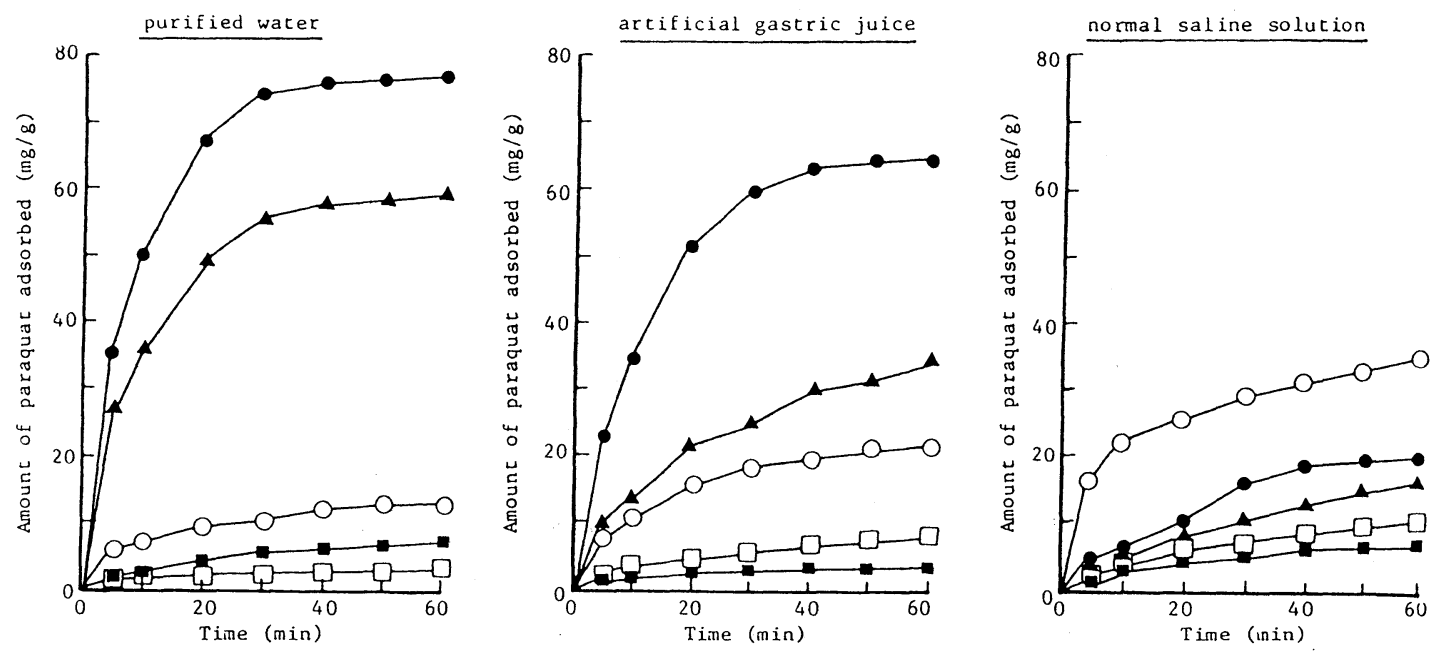

Fig. 2 Time courses of amounts of paraquat adsorbed onto chitosan beads and activated carbon

: $\mathrm{CM}, \boldsymbol{\Delta}: \mathrm{SU}$

: CS, $\square$ : BCW, $\bigcirc$ : activated carbon 
activated carbon were successively larger in the order of normal saline solution $>$ in artificial gastric juice $>$ in purified water.

In addition, the effect of the sodium chloride concentration in the solution on the removal of paraquat by chitosan was investigated. The time courses of the amounts of paraquat adsorbed in various saline solutions are shown in Figure 3. The concentrations of sodium chloride in the paraquat solution were $0,0.45,0.9$ and $1.8 \%$. Addition of sodium chloride showed no effect on the absorbance at $600 \mathrm{~nm}$ as measured by colorimetric determination of the paraquat concentration.
With the increase of sodium chloride concentration, the amounts of paraquat adsorbed onto $\mathrm{CM}$ and SU were decreased. No ef fect of sodium chloride concentration on the removal of paraquat by CS was recognized. However, with the increase of sodium chloride concentration, the amount adsorbed onto BCW was increased.

It was hence concluded that paraquat was easily adsorbed onto CM and SU in purified water, but that paraquat removal was inhibited by sodium chloride addition. The paraquat removal by BCW and activated carbon was accelerated with the increase of the sodium chloride concen-
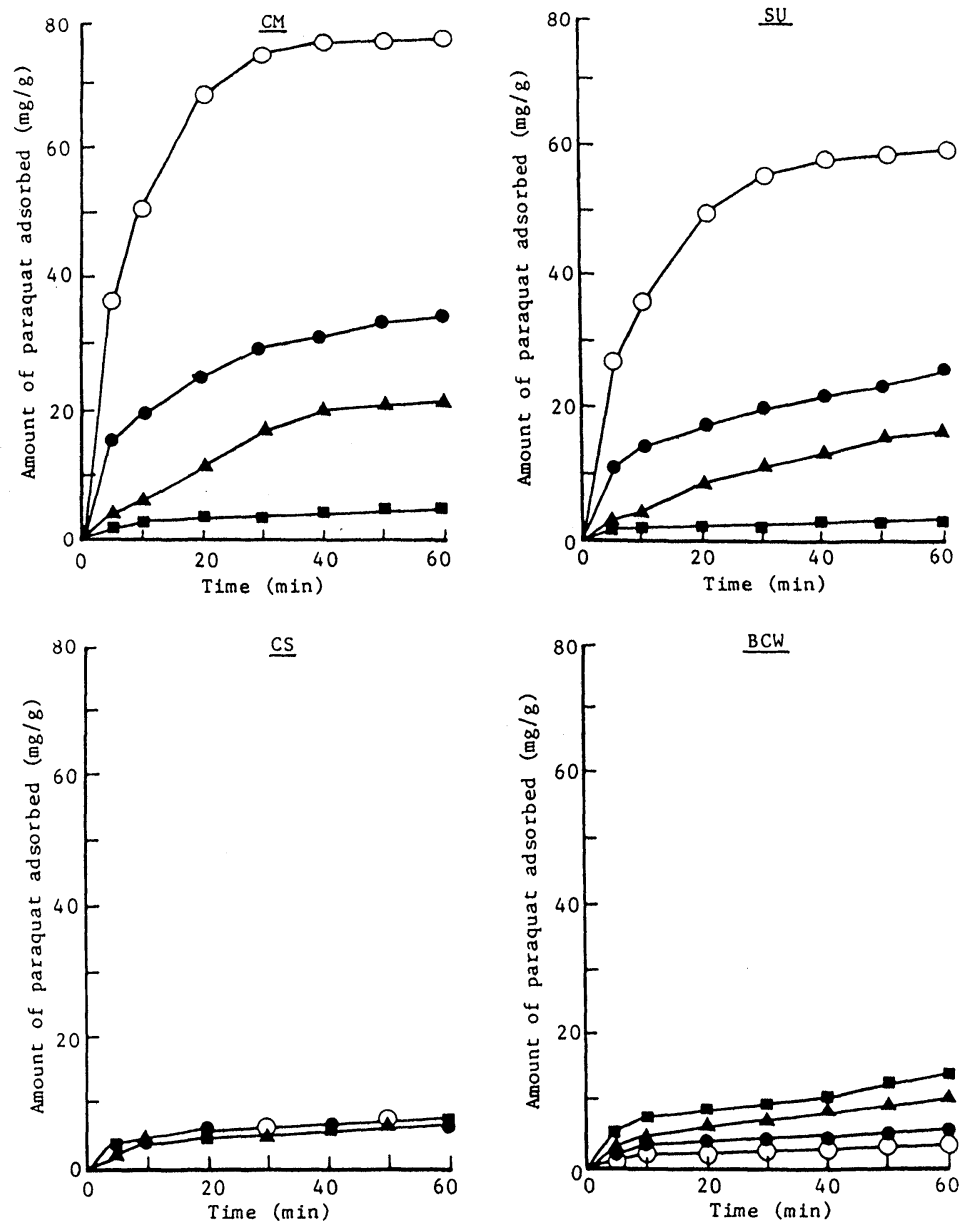

Fig. 3 Time courses of amounts of paraquat adsorbed in solutions of different sodium chloride concentrations

$\mathrm{NaCl} ; \bigcirc: 0 \%$

: $0.45 \%$,

: $0.9 \%$,

$: 1.8 \%$ 
tration in the solution.

\section{Discussion}

For the primary treatment of acute drug poisoning, activated carbon has been used, and its efficacy has been reported ${ }^{4,19,20}$. The efficacy of some adsorbents for paraquat poisoning has been also reported ${ }^{4 \sim 9)}$. However, little study on the evaluation of chitosan, which is a safe biopolymer for the organism and environment, for paraquat poisoning has been done so far. Although activated carbon has been evaluated as a safe and effective gastrointestinal adsorbent, the removal capacity of $\mathrm{CM}$ and $\mathrm{SU}$ chitosan in purified water for paraquat is inferior to that of activated carbon ${ }^{21)}$. Paraquat removal by activated carbon is mostly based on physical adsorption, whereas that by CM and SU chitosan appered to occur via ion exchange and/or chemisorption because the paraquat removal by these chitosan beads was inhibited in saline solutions. Kayexalate and kalimate are medical cation exchange resins for the treatment of hyperkalemia, and these resins are noted as a new antidote for paraquat poisoning ${ }^{8,9)}$. However, the the capacity to remove paraquat of these resins in easily inhibited by the presence of saline solution ${ }^{22)}$. That of chitosan in also inhibited by the presence of sodium chloride in the solution. Therefore, the inhibitory effect of saline solution on the paraquat removal by $\mathrm{CM}$ and $\mathrm{SU}$ chitosan must be considered. On the other hand, the paraquat removal by $\mathrm{BCW}$ chitosan was accelerated in the presence of sodium chloride. The enhancing effect of saline on the paraquat adsorption capacity of activated carbon has been reported. Honda et $a l^{23)}$ reported that the amount of paraquat adsorbed onto activated carbon was larger in normal saline solution than in artificial gastric juice or in purified water. We reported that the amount of paraquat adsorbed onto activated carbon was increased with the increase of the sodium chloride concentration in the solution ${ }^{21)}$. It is not clear why the acceleration of paraquat adsorption onto activated carbon occurs with the addition saline. The dissociation of paraquat in solution was inhibited by the addition of saline, that is, salting out takes place in the solution. Therefore, it is considered that paraquat is adsorbed more abundantly onto activated carbon. The change in surface charge caused by the presence of sodium chloride can not be neglected. In saline adsorption onto activated carbon, anions are specifically adsorbed. Then an electrical double layer is formed on the surface of the adsorbent, and attracts cations in solution ${ }^{24)}$. It is assumed that paraquat cations are more attracted to the electrical double layer than sodium cations in adsorption onto chitosan beads. Therefore, it is considered that paraquat removal is accelerated by the addition of sodium chloride.

Chitosan has three functional groups, amino-, primary hydroxyl- and secondary hydroxyl groups, in its structure. The $\mathrm{C}_{6}$ hydroxyl group in CM and SU is substituted for by carboxymethyl and sulfonic groups, respectively. These substituted groups are negatively charged, and paraquat is dissociated into cations in solution . Therefore, it is assumed that the high removal capacity of $\mathrm{CM}$ and SU for paraquat in purified water is due to the electrostatic interaction between the negatively charged substituted group of chitosan beads and paraquat cations.

\section{References}

1) Ukai, S. and Kawase, S.: Paraquat poisoning and forensic chemistry, Eisei Kagaku, 31, 283-297 (1985). (in Japanese with English abstract)

2 ) Swan, A.A.B. : Exposure of spray operators to paraquat, Br. J. Ind. Med. , 26, 322-329 (1968).

3 ) Howard, J.K.: Paraquat, a review of worker exposure in normal usage, J. Soc. Occup. Med. , 30, 6-11 (1980).

4 ) Okonek, S., Weilemann, L.S. , Majdandzic, J., Setyadharma, H., Reinecke, H.J., Baldamus, C.A., Lohmann, J., Bonzel, 
K.E. and Thon, T. : Successful treatment of paraquat poisoning; activated charcoal per os and "continuous hemoperfusion", J. Toxicol. Clin. Toxicol. , 19, 807-819 (198283) .

5) Kawai, M., Koyama, M., Kaneko, Y. and Ogasawara, S.: Efficacy of the use of adsorbents in the treatment of paraquat poisoning of dog, J. Jpn. Assoc. Rural Med., 30, 791-802 (1981) . (in Japanese with English abstract)

6 ) Smith, L.L., Wright, A., Wyatt, I. and Rose, M.S. : Effective treatment for paraquat poisoning in rats and its relevance to the treatment of paraquat poisoning in man, Br. Med. J. , 4, 569-571 (1974).

7 ) Donald, C.S. , Gordon, K.I. and Walter, C.F.: Screening of various adsorbents for protection against paraquat poisoning, Bull. Environ. Contam. Toxicol., 10, 193199 (1973) .

8 ) Nokata, M., Tanaka, T., Tsuchiya, K. and Yamashita, M.: Alleviation of paraquat toxicity by kayexalate and kalimate in rats, Acta Pharmacol. Toxicol. , 55, 158-160 (1984).

9) Yamashita, M., Naito, H. and Takagi, S.: The effectiveness of a cation resin (kayexalate) as an adsorbent of paraquat, experimental and clinical studies, Hum. Toxicol. , 6, 89-90 (1987).

10) Yoshida, H.: Application of chitin and chitosan, Kagaku to Kogyo (Osaka), 64, 325330 (1990). (in Japanese)

11) Gonzalez-Davila, M., Santana-Casiano, J.M. and Millero, F.J.: The adsorption of $\mathrm{Cd}$ (II) and $\mathrm{Pb}$ (II) to chitin in seawater, $\mathrm{J}$. Colloid Interface Sci. , 137, 102-110 (1990).

12) McKay, G., Blair, H.S. and Gardner, J.: Rate studies for the adsorption of dyestuffs onto chitin, J. Colloid Interface Sci. , 95, 108-119 (1983).

13) Yoshida, H., Fukuda, S., Okamoto, A. and Kataoka, T. : Recovery of direct dye and acid dye by adsorption on chitosan fiber, equilibria, Wat. Sci. Tech., 23, 1667-1676 (1991).

14) Hou, W.M., Miyazaki, S., Takada, M. and Komai, T.: Sustained release of indomethacin from chitosan granules, Chem . Pharm. Bull., 33, 3986-3992 (1985).

15) Sawayanagi, Y., Nambu, N. and Nagai, T. : Enhancement of dissolution properties of griseof ulvin from ground mixtures with chitin or chitosan, Chem. Pharm. Bull., 30, 4464-4467 (1982).

16) Lin, S.Y. and Perng, R.I. : Adsorption and desorption of indomethacin on cellulose-like biopolymers: chitin and chitosan, Chem . Pharm. Bull., 40, 1058-1060 (1992).

17) Calderbank, A. and Yuen, S.H.: An ionexchange method for determining paraquat residues in food crops, Analyst, 90, 99-106 (1965) .

18) Nippon Koteisho Kyokai (Supervised): The Japanese Pharmacopoeia, 11th edition, B349-351, Hirokawa Syoten, Tokyo (1986). (in Japanese)

19) Javaid, K.A. and El-Mabrouk, B.H.: In vitro adsorption of phenobarbital onto activated carbon, J. Pharm. Sci., 72, 82-85 (1983) .

20) Neuvonen, P.J., Kannistro, H. and Hirvisalo, E.L.: Effect of activated charcoal on absorption of tolbutamide and valproate in man, Eur. J. Clin. Pharmacol., 24, 243246 (1983).

21) Tanada, S. , Nakamura, T., Xiaohong, M., Higuchi, T., Miyoshi, T., Tamura, T. and Imaki, M. : Enhancing effect of sodium chloride on in vitro adsorption of paraquat onto activated carbon, Jpn. J. Hyg . , 46, 10511056 (1992).

22) Nakamura, M., Tanada, S., Nakamura, T. , Keshi, H. and Kawanishi, T. : In vitro study on the inhibitory effect of magnesium cation on paraquat removal by medical cation exchange resin, Bull. Environ. Contam. 
Jpn. J. Hyg. , Vol. 48, No. 5, December 1993

Toxicol. , 45, 165-169 (1990).

23) Honda, Y., Nakano, M. and Nakano, N.: Effects of several salts on in vitro adsorption of paraquat by cation exchange resins and activated carbons, Jpn. J. Hosp. Pharm. , 18, 100-105 (1992). (in Japanese with English abstract)

24) Urano, K., Sonai, M., Nakayama, R. and
Kobayashi, Y.: Adsorption mechanism of strong acids, strong bases or their salts by activated carbon and $\mathrm{pH}$ of the solution with carbon suspension, Nippon Kagaku Kaishi, 1976, 1773-1778 (1976). (in Japanese with English abstract)

(Received Mar. 25, 1993/Accepted Sep. 27, 1993) 